\title{
Prescription Patterns for Bipolar Disorder in Asian Countries: Findings from Research on Asian Prescription Pattern-Bipolar Disorder
}

\author{
Shih-Ku Lin ${ }^{1,2}$, Shu-Yu Yang ${ }^{3}$, Seon-Cheol Park ${ }^{4}$, Ok-jin Jang ${ }^{5}$, Xiaomin Zhu ${ }^{6}$, Yu-Tao Xiang ${ }^{7,8}$, \\ Wen-Chen Ouyang ${ }^{9,10,11}$, Afzal Javed ${ }^{12}$, M. Nasar Sayeed Khan ${ }^{13}$, Sandeep Grover ${ }^{14}$, Ajit Avasthi ${ }^{14}$, \\ Roy Abraham Kallivayaliil ${ }^{15}$, Kok Yoon Chee ${ }^{16}$, Norliza Chemi ${ }^{17}$, Takahiro A. Kato ${ }^{18}$, Kohei Hayakawa ${ }^{18}$, \\ Pornjira Pariwatcharakul ${ }^{19}$, Margarita Maramis ${ }^{20}$, Lakmi Seneviratne ${ }^{21}$, Sim Kang ${ }^{22}$, Wai Kwong Tang ${ }^{23}$, \\ Tin $\mathrm{Oo}^{24}$, Norman Sartorius ${ }^{25}$, Chay-Hoon Tan ${ }^{26}$, Mian-Yoon Chong ${ }^{27}$, Yong Chon Park ${ }^{4}$, Naotaka Shinfuku ${ }^{28}$ \\ ${ }^{1}$ Department of Psychiatry, Taipei City Hospital and Psychiatric Center, Taipei, Taiwan, ${ }^{2}$ Department of Psychiatry, Chang Gung Memorial \\ Hospital at Linkou, Taoyuan, Taiwan, ${ }^{3}$ Department of Pharmacy, Taipei City Hospital, Taipei, Taiwan, ${ }^{4}$ Department of Neuropsychiatry, Hanyang \\ University Guri Hospital, Guri, Korea, ${ }^{5}$ Department of Psychiatry, Bugok National Hospital, Changyeong, Korea, ${ }^{6}$ Department of Psychiatry, \\ Suzhou Guangji Hospital, the Affiliated Guangji Hospital of Soochow University, Suzhou, China, ${ }^{7}$ Department of Psychiatry, Beijing Anding \\ Hospital of Capital Medical University, Beijing, China, ${ }^{8}$ Unit of Psychiatry, Faculty of Health Sciences, University of Macau, Macao SAR, China, \\ ${ }^{9}$ Department of Geriatric Psychiatry, Jianan Psychiatric Center, Tainan, Taiwan, ${ }^{10}$ Department of Psychiatry, Kaohsiung Medical University, \\ Kaohsiung, Taiwan, ${ }^{11}$ Department of Nursing, Shu-Zen Junior College of Medicine and Management, Kaohsiung, Taiwan, ${ }^{12}$ Pakistan Psychiatric \\ Research Centre, Fountain House, Lahore, Pakistan, ${ }^{13}$ Department of Psychiatry, Services Hospital, Lahore, Pakistan, ${ }^{14}$ Department of Psychiatry, \\ Post Graduate Institute of Medical Education and Research, Chandigarh, India, ${ }^{15}$ Pushpagiri Institute of Medical Sciences, Tiruvalla, India, \\ ${ }^{16}$ Department of Psychiatry \& Mental Health, Tunku Abdul Rahman Institute of Neurosciences, Kuala Lumpur Hospital, Kuala Lumpur, Malaysia, \\ ${ }^{17}$ Department of Psychiatry and Mental Health, Hospital Kajang, Selangor, Malaysia, ${ }^{18}$ Department of Neuropsychiatry, Graduate School of \\ Medical Sciences, Kyushu University, Fukuoka, Japan, ${ }^{19}$ Faculty of Medicine Siriraj Hospital, Mahidol University, Bangkok, Thailand, ${ }^{20}$ Department \\ of Psychiatry, Dr. Soetomo Hospital - Faculty of Medicine, Airlangga University, Surabaya, Indonesia, ${ }^{21}$ Department of Psychiatry, University of \\ Sri Jayewardenepura, Nugegoda, Sri Lanka, ${ }^{22}$ Institute of Mental Health, Buangkok Green Medical Park, Singapore, ${ }^{23}$ Department of Psychiatry, \\ Chinese University of Hong Kong, Hong Kong, China, ${ }^{24}$ Mental Health Hospital, Yangon University of Medicine, Yangon, Myanmar, \\ ${ }^{25}$ Association for the Improvement of Mental Health Programs, Geneva, Switzerland, ${ }^{26}$ Department of Pharmacology, National University of \\ Singapore, Singapore, ${ }^{27}$ Department of Psychiatry, Kaohsiung Chang Gung Memorial Hospital, Kaohsiung \& Chang Gung University School \\ of Medicine, Linkou, Taiwan, ${ }^{28}$ School of Human Sciences, Seinan Gakuin University, Fukuoka, Japan
}

Objective: Pharmacotherapy including mood stabilizers and antipsychotics are frequently used in bipolar disorder (BD); however, the lack of consensus regarding the definition of polypharmacy hinders conducting comparative studies across different settings and countries. Research on Asian Prescription Pattern (REAP) is the largest and the longest lasting international collaborative research in psychiatry in Asia. The objective of REAP BD was to investigate the prescription patterns of psychotropic medications across Asian countries. The rates of polypharmacy and psychotropic drug load were also analyzed.

Methods: The data collection was web-based. Prescription patterns were categorized as (1) mood stabilizer monotherapy: one mood stabilizer; (2) antipsychotic monotherapy: one antipsychotic; (3) simple polypharmacy: one mood stabilizer and one antipsychotic; and (4) complex polypharmacy: $\geq 2$ mood stabilizers or/and antipsychotics. The psychotropic drug load in each patient was calculated using the defined daily dose method.

Results: Among 2003 patients with BD (52.1\% female, 42.4 years) from 12 countries, 1,619 (80.8\%) patients received mood stabilizers, 1,644 (82.14\%) received antipsychotics, and $424(21.2 \%)$ received antidepressants, with $14.7 \%$ mood stabilizer monotherapy, $13.4 \%$ antipsychotic monotherapy, $48.9 \%$ simple polypharmacy, 20.3\% complex polypharmacy, and $2.6 \%$ other therapy. The average psychotropic drug load was $2.05 \pm 1.40$. Results varied widely between countries.

Received: October 15, 2020 / Revised: December 25, 2020 / Accepted: December 26, 2020

Address for correspondence: Shih-Ku Lin

Department of Psychiatry, Taipei City Hospital and Psychiatric Center, 309 Songde Road, Taipei 110, Taiwan

E-mail: sklin@tpech.gov.tw

ORCID: https://orcid.org/0000-0002-5123-0389

(c) This is an Open-Access article distributed under the terms of the Creative Commons Attribution Non-Commercial License (http://creativecommons.org/licenses/by-nc/4.0) which permits unrestricted non-commercial use, distribution, and reproduction in any medium, provided the original work is properly cited. 
Conclusion: Over $70 \%$ of psychotropic regimens involved polypharmacy, which accords with the high prevalence of polypharmacy in BD under a permissive criterion (2 or more core psychotropic drugs) worldwide. Notably, $\geq 80 \%$ of our sample received antipsychotics, which may indicate an increasing trend in antipsychotic use for BD treatment.

KEY WORDS: Bipolar disorder; Polypharmacy; Psychotropic drug load; Antipsychotics; Mood stabilizers.

\section{INTRODUCTION}

Bipolar disorder (BD) is a severe, recurring mental illness. Pharmacotherapy is crucial in the treatment of BD, supplemented by social and psychological interventions. Pharmaceutical treatments for BD are mood stabilizers and antipsychotics, which are sometimes combined with anxiolytics and hypnotics. In certain situations, patients are prescribed antidepressants. The movement side effects of second-generation antipsychotics (SGAs) are relatively minor; thus, they have even become the principal therapeutic agents employed to replace mood stabilizers $[1,2]$. Long-acting injection antipsychotics also have become more common [3]. The symptoms or signs and the course of BD are complicated, dynamic, and changeable regarding the illness course and phase. Furthermore, medication use differs in each phase. Despite the availability of numerous pharmacological textbooks and treatment guidelines for a specific disorder [4,5], wide variations exist in selecting drugs and dosages between different physicians and also different countries [6,7].

Polypharmacy-related studies are much less popular for BD than for schizophrenia [8,9]; especially the definitions of polypharmacy in BD are vague and inconsistent. In a review, Fornaro et al. [10] discussed the definition of polypharmacy in BD; most definitions of polypharmacy centered on the use of 2 or more psychotropic drugs at once, and complex polypharmacy centered on the use of $\geq 4$ psychotropic and non-psychotropic drugs at once. This type of classification is easily applicable in clinical practice but not feasible for scientific research because only mood stabilizers and antipsychotics are currently indicated for BD. Patients receiving antidepressants, anxiolytics, or antihypertensives should not be included in polypharmacy.

Research on Asian Prescription Patterns (REAP) is an international collaborative consortium for studying the prescription patterns of psychotropic drugs across countries (http://reap.asia/index.html). The aim of this study (REAP-BD) was to survey prescription patterns for $\mathrm{BD}$ in Asian countries. Prescriptions for mood stabilizers, antipsychotics, and other psychotropic drugs were included in the investigation of prescription patterns to reveal the rate of polypharmacy, the dosage of each class of drug, the different proportions of mood stabilizers, the rate of first-generation antipsychotics (FGAs) and SGAs, and other combined medications.

\section{METHODS}

\section{Design and Participants}

A convenience sampling method was used to enroll study patients. Patients with BD who were undergoing pharmacotherapy could be included in the survey. Participating psychiatrists assessed patients according to their clinical features and assigned them a status based on ICD-10-CM codes F31.0 to F31.9. An online website-based data key-in system was used for data collection. The research protocol can be accessed at http://www.reap.asia/ pdf/reap_bd_protocol.pdf. In brief, data regarding daily medications prescribed for treating inpatients or outpatients with BD, including mood stabilizers, antipsychotics, antidepressants, anxiolytics, hypnotics, and other concomitant medications, as well as demographics and laboratory tests, were collected.

\section{Classification of Polypharmacy in BD}

Because mood stabilizers and some antipsychotics are indicated for treating BD, defining polypharmacy is complex. In the present study, core medications for BD were defined as mood stabilizers and antipsychotics, and other psychotropic drugs, such as antidepressants, hypnotics, and anxiolytics, were categorized as peripheral medications. The most commonly accepted definition of polypharmacy in schizophrenia is the simultaneous use of 2 or more antipsychotics [9]; thus, we proposed polypharmacy in BD as the simultaneous use of 2 or more core medications. To simplify operational criteria, prescription patterns for BD monotherapy and polypharmacy were categorized as follows: (1) mood stabilizer monotherapy: 
only one mood stabilizer used; (2) antipsychotic monotherapy: only one antipsychotic used; (3) simple polypharmacy: one mood stabilizer and one antipsychotic used; and (4) complex polypharmacy: $\geq 2$ mood stabilizers or/and antipsychotics used. The use of long-acting injectable antipsychotics will be categorized as a type of antipsychotic. The use of antidepressants, anxiolytics and hypnotics was also recorded, and was classified as combined medications.

\section{Psychotropic Drug Load to Indicate the Dosage Used}

Conventionally, a chlorpromazine equivalent is used to compare the therapeutic dose of an antipsychotic $[11,12]$. Since the core medications for $\mathrm{BD}$ including antipsychotic and mood stabilizers, the Anatomical Therapeutic Chemical (ATC) Classification System (i.e., the ATC/DDD [defined daily dose] Index 2016 [accessed May 1, 2019]) [13], was employed to calculate the drug load to compare the dosage used across the different categories of psychotropic drugs. For instance, the mood stabilizer load was calculated using the sum of the prescribed daily dose of each mood stabilizer divided by its DDD. The value obtained reflected the quantity of mood stabilizer received by a patient, and the psychotropic drug load (PDL) was used to represent the psychotropic medication (antipsychotics, mood stabilizers, antidepressants, anxiolytics and hypnotics) quantity prescribed to treat a mental disorder. For lithium, the suggested DDD was $24 \mathrm{mmol}$, where lithium carbonate $\left(\mathrm{Li}_{2} \mathrm{CO}_{3}\right)$ weight is $73.891 \mathrm{~g} / \mathrm{mol}$. As 73.891 $\mathrm{g} / \mathrm{mol} \times 24 \mathrm{mmol} / 2=886.692 \mathrm{mg}$, in the present study, $900 \mathrm{mg}$ was used as the DDD for convenient calculation.

This study was sponsored by the Ministry of Science and Technology of Taiwan (106-2314-B-532-009) and approved by the Taipei City Hospital Research Ethics Committee (TCHIRB: 10605117-E).

\section{RESULTS}

In total, 2003 patients with BD (70.3\% outpatients; $52.1 \%$ female) were enrolled from 13 countries or regions. The numbers and demographics of each country are presented in Table 1, listed in the order of the number of patients enrolled. The mean age was $42.4 \pm 15.2$ years, with the oldest patients in Singapore (56.2 \pm 15.5$)$ and the youngest in Myanmar $(30.8 \pm 9.0)$. The mean body weight was $67.1 \pm 14.0 \mathrm{~kg}$, with the highest patient weights in

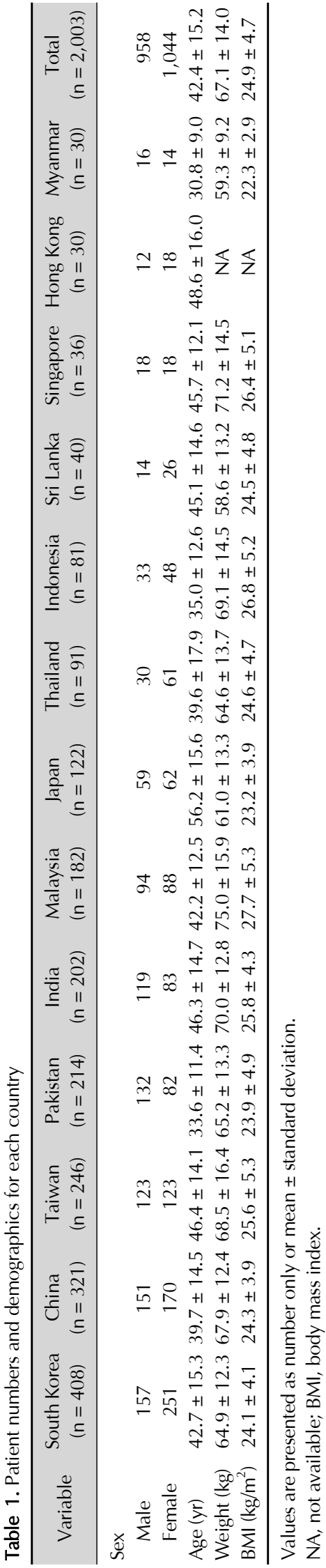



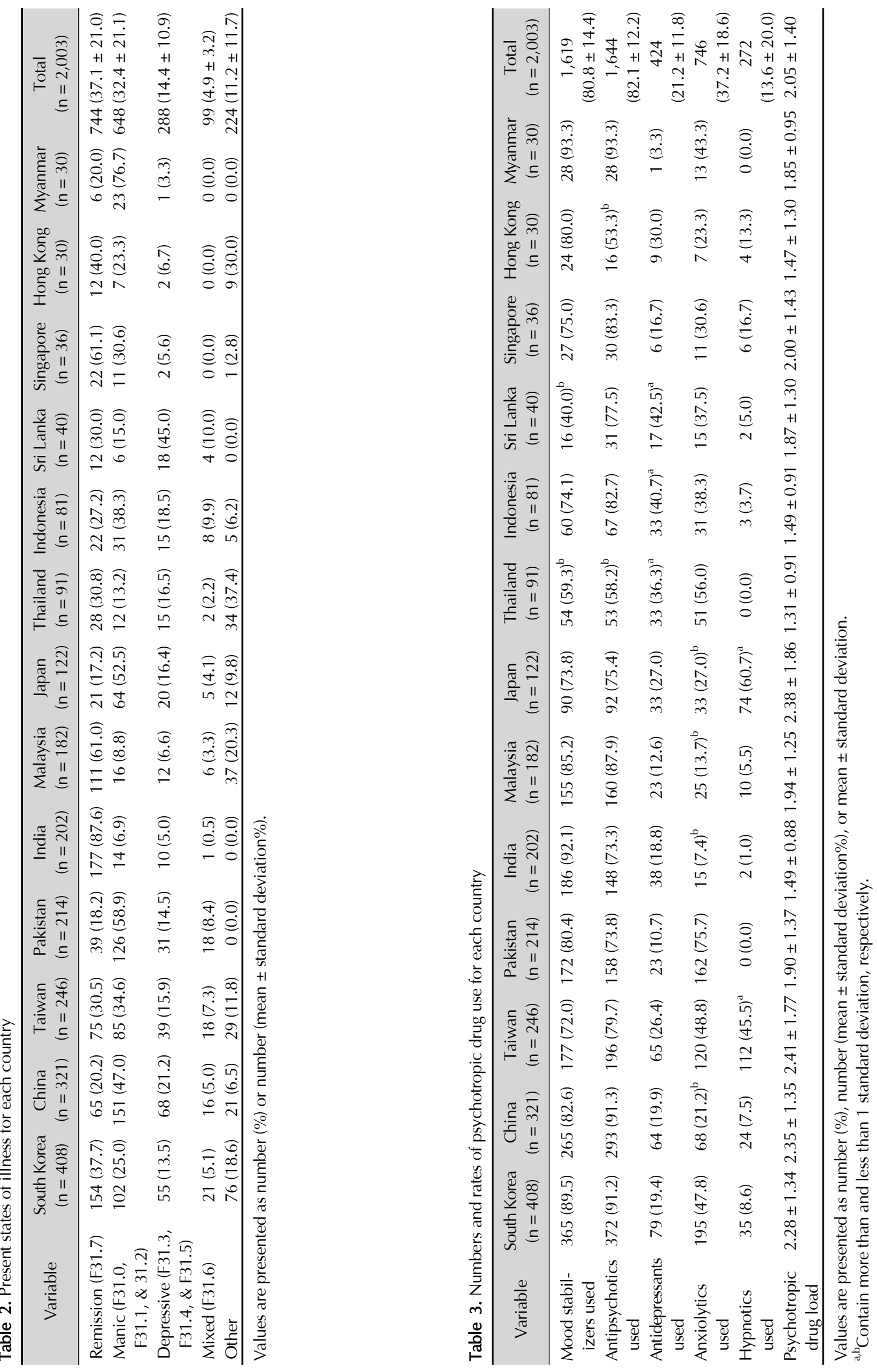
Malaysia (75.0 \pm 15.9$)$ and the lowest in Sri Lanka (58.6 \pm 13.2). The mean body mass index was $24.9 \pm 4.7$. Table 2 summarizes the stages of illness at enrollment. The largest proportion of patients were in the remission phase (37.1\%), followed by manic (32.4\%), depressed (14.4\%), others $(11.2 \%)$, and mixed (4.9\%). Regarding the illness course pattern, $43 \%$ of patients presented with mania or hypomania, followed by depression and a euthymic interval (MDI), and $30 \%$ of patients presented with depression, followed by mania or hypomania (DMI). The remaining participants exhibited unknown patterns. Table 3 illustrates the numbers and rates of psychotropic drugs used. Over $80 \%$ of patients were prescribed mood stabilizers or antipsychotics, $21.2 \%$ antidepressants, $37.2 \%$ anxiolytics, and $13.6 \%$ hypnotics. Valproic acid (47.2\%) was the most prescribed mood stabilizer, followed by lithium (28.5\%), carbamazepine $(6.7 \%)$, and lamotrigine $(1.1 \%)$, and most prescribed antipsychotics were quetiapine (33.1\%), olanzapine (19.6\%), risperidone (14.4\%), and aripiprazole (11.3\%). The total PDL was $2.05 \pm 1.40$, with a median drug load of 0.67 for mood stabilizers, 0.8 for SGAs, 0.50 for FGAs, 0.40 for anxiolytics, and 1.00 for hypnotics.

Table 4 presents the prescription pattern across countries. The most common prescription patterns were simple polypharmacy $(48.9 \% \pm 15.8 \%)$, followed by complex polypharmacy $(20.3 \% \pm 10.1 \%)$, mood stabilizer monotherapy $(14.7 \% \pm 10.2 \%)$, antipsychotic monotherapy $(13.4 \% \pm 10.0 \%)$, and antidepressant monotherapy $(2.1 \% \pm$ $4.0 \%)$. Only 11 patients received neither mood stabilizers nor antipsychotics. Table 5 compares the psychotropic drug use and prescription pattern between different BD phases (diagnoses F31.8 and F31.9 excluded, $n=1,779$ ).

\section{DISCUSSION}

This is the first comparative study of prescription patterns in patients with BD across Asian countries. The number of participants and course phases differed considerably between countries; thus, the entire group was first analyzed and results were then compared between countries.

Notably, approximately $82.1 \%$ of patients were receiving antipsychotics as the first choice of medications (67.6\% of SGA, $6.9 \%$ of FGA, and $7.6 \%$ of both). No differences were noted in the use of antipsychotics between different illness phases, except slightly less in depressive 
Table 5. Comparison of psychotropic drug use and prescription patterns between different bipolar disorder phases

\begin{tabular}{lccccc}
\hline \multicolumn{1}{c}{ Variable } & $\begin{array}{c}\text { Remission } \\
(\mathrm{n}=744)\end{array}$ & $\begin{array}{c}\text { Manic } \\
(\mathrm{n}=648)\end{array}$ & $\begin{array}{c}\text { Depressive } \\
(\mathrm{n}=288)\end{array}$ & $\begin{array}{c}\text { Mixed } \\
(\mathrm{n}=99)\end{array}$ & $\begin{array}{c}\text { Total } \\
(\mathrm{n}=1,779)\end{array}$ \\
\hline Mood stabilizers & $628(84.4)$ & $553(85.3)$ & $189(65.6)$ & $75(75.8)$ & $1,445(81.2)$ \\
Antipsychotics & $598(80.4)$ & $557(86.0)$ & $222(77.1)$ & $90(90.9)$ & $1,467(82.5)$ \\
Antidepressants & $136(18.3)$ & $53(8.2)$ & $142(49.3)$ & $30(30.3)$ & $361(20.3)$ \\
Anxiolytics & $175(23.5)$ & $272(42.0)$ & $144(50.0)$ & $54(54.5)$ & $645(36.3)$ \\
Hypnotics & $66(8.9)$ & $98(15.1)$ & $59(20.5)$ & $16(16.2)$ & $239(13.4)$ \\
Simple polypharmacy & $397(53.4)$ & $340(52.5)$ & $108(37.5)$ & $45(45.5)$ & $890(50.0)$ \\
Complex polypharmacy & $118(15.9)$ & $156(24.1)$ & $46(16.0)$ & $29(29.3)$ & $349(19.6)$ \\
Mood stabilizer monotherapy & $130(17.5)$ & $78(12.0)$ & $49(17.0)$ & $7(7.1)$ & $264(14.8)$ \\
Antipsychotic monotherapy & $86(11.6)$ & $64(9.9)$ & $72(25.0)$ & $17(17.2)$ & $239(13.4)$ \\
Antidepressant monotherapy & $12(1.6)$ & $5(0.8)$ & $11(3.8)$ & $1(1.0)$ & $29(1.6)$ \\
Neither & $1(0.1)$ & $5(0.8)$ & $2(0.7)$ & $0(0.0)$ & $8(0.4)$ \\
\hline
\end{tabular}

Values are presented as number (\%).

phase (Table 5). The high rate of use of antipsychotics in the treatment of $\mathrm{BD}$ is similar to rates reported in Western countries. Hayes et al. [14] reported an increased prescription rate of antipsychotics from $14.2 \%$ to $41.9 \%$ from 1995 to 2009 in a very large British BD database. During this period, the use of SGA increased dramatically from $0 \%$ to $35 \%$. A 17-year observational study in the United States reported that the prescription rate of antipsychotic medications per 100 persons increased from 3.25 to 6.18 among outpatients with $\mathrm{BD}$, with SGA medications comprising the majority [15]. A 25-year Finnish nationwide cohort study of 18,018 patients with BD reported that $81.1 \%$ of participants took antipsychotics during follow-up [16]. Antipsychotics have primarily been used in manic or depressive phases with psychotic features in the past; however, an increasing number of antipsychotics, especially SGAs, are indicated for BD. Treatment guidelines for BD have suggested antipsychotics as the first-line choice of medication [17-19]. Several possible reasons exist for the increasing trend of antipsychotic prescription for patients with BD. First, SGAs with a lower propensity for extrapyramidal symptoms and the same or higher efficacy than typical antipsychotics have been introduced $[20,21]$. Second, psychiatric medications have become more acceptable in public opinion over the last 2 decades; thus, patients with $\mathrm{BD}$ are increasingly willing to use medications for depression, panic attacks, or other psychiatric problems to improve their quality of life [22]. Third, the role of psychotherapy appears to have become less prominent based on the declining number of psychiatrists specializing in psychotherapy and the decreased number of patients receiving psychotherapy over the last 2 decades [22,23].

Mood stabilizers are the gold standard for BD, both in acute and maintenance therapy. Our results also indicated that over $85 \%$ of patients were prescribed mood stabilizers in remission and manic phases, and less in mixed $(75.8 \%)$ and depressive (65.6\%) phases. For antipsychotics, more mixed and manic phases (> 85\%) were prescribed and less in remission and depressive phased (Table 5).

Because of vague and inconsistent definitions, comparing the rates of polypharmacy in BD between studies is difficult. A post hoc report based on the STEP-BD study ( $\mathrm{n}=$ 4,035 ) revealed that $21 \%$ of patients used 1 core medication (monotherapy, lithium, anticonvulsants, antidepressants, and antipsychotics), $28 \%$ used 2 (simple polypharmacy), $22 \%$ used 3 , and $18 \%$ used 4 (complex polypharmacy); $12 \%$ did not use any core medications [24]. A comparative effectiveness trial of 482 patients with $\mathrm{BD}$ indicated that $43 \%$ had received complex polypharmacy over the study ( $\geq 4$ types of lithium, antipsychotics, antidepressants, anticonvulsants, or anxiolytics) [25]. The authors concluded that patients with $\mathrm{BD}$ receiving complex polypharmacy were less likely to adhere to therapy and were less likely to achieve remission. A survey conducted in Poland in 127 patients with BD showed that 78 (61\%) patients were prescribed 2 medications, 27 (21.3\%) were prescribed 3 , and one was prescribed 4 (mood stabilizers or SGA), with the combination of mood stabilizers and SGA was most commonly used $(n=61,48 \%$ ) [26]. Here we suggest that using the classification system defined in 
this study, namely only mood stabilizer and antipsychotic regarded as core medication for $\mathrm{BD}$, would enable easier comparison of the polypharmacy rate in BD between different studies and different phases (Table 5). By this definition, our results revealed more than $50 \%$ of simple polypharmacy was prescribed in remission and manic phase; and complex polypharmacy most popular in mixed phase $(29.3 \%)$, mood stabilizer monotherapy least in mixed phase. It is interesting to note that antipsychotic monotherapy was most popular in depressive phase, followed by mixed phase. What is the ideal proportion of prescription pattern needs further investigation.

The majorities of treatment guidelines do not recommend antidepressants in BD, even in the depressed phase, or specify that they should be used with caution $[18,19,27]$. Lyall et al. [28] examined the trends of psychotropic drug use in a cohort of 23,135 patients with BD between 2009 and 2016 in Scotland and determined that the most common form of treatment was antidepressant monotherapy (24.96\%), followed by antipsychotic monotherapy $(12.94 \%)$, with only $5.90 \%$ of treated patients receiving lithium monotherapy. The authors concluded that their findings represented a gap between treatment guidelines and clinical practice, which was a cause of concern. Haeberle et al. [29], investigated a cohort of inpatients with bipolar depression ( $n=2,246$; 1994-2009) and reported that $85 \%$ of all patients received more than one class of psychotropic drugs; $74 \%$ received antidepressants in combination therapy, 55\% received antipsychotics, $48 \%$ received anticonvulsants, and $33 \%$ received lithium. The rate of antidepressant use in our sample was $21.2 \%$, and approximately half of them $(49.3 \%)$ were in a depressed phase (Table 5). The rate of antidepressant monotherapy was only $2.1 \%$ (Table 4 ). These figures are more compatible with most treatment guidelines. Our results revealed that more females $(22.9 \%)$ than males $(19.2 \%)$ were prescribed antidepressants, which is in accordance with the findings of a study conducted by Weinstock et al. [30] (27\% vs. $11 \%)$.

Our findings revealed differences in psychotropic drug prescription preferences between countries (Table 3). For instance, fewer mood stabilizers were prescribed in Thailand and Sri Lanka (less than one standard deviation), and fewer antipsychotics were prescribed in Thailand and Hong Kong, whereas more antidepressants were prescribed in Thailand, Indonesia, and Sri Lanka (more than one standard deviation). Interestingly, more hypnotics were prescribed in Taiwan and Japan compared with other countries. This preference for hypnotic prescriptions is also reported in the survey of schizophrenia in these 2 countries [31]. Regarding prescription patterns (Table 4), China and Myanmar had more simple polypharmacy by one standard deviation, whereas Korea and Japan had more complex polypharmacy. India and Hong Kong had more mood stabilizer monotherapy, whereas Indonesia and Sri Lanka had more antipsychotic monotherapy. Although the rate is still low (13.2\%), Thailand had the most antidepressant monotherapy. Regarding PDL, Korea, China, Taiwan, and Japan prescribed higher doses of psychotropics than did other countries. For individual psychotropics drugs, only the load of hypnotics reached 1.0, and all others were below the suggested daily dose. This might have been because approximately $70 \%$ of patients received either simple or complex polypharmacy. Most studies have investigated the types of psychotropic drugs used but did not compare the dosage. By the application of PDD/DDD, it would be easier to compare the dosage of each psychotropic drug, or PDL in total. Different prescription pattern and dosage between countries might be attributed to psychiatrists' different training backgrounds, the availability of medications, civil culture, and the health insurance system of each country. Therefore, this topic warrants further investigation.

Tondo et al. [32] studied the major course pattern in 1,130 patients with BD and determined that $56.8 \%$ of patients could be characterized for major course patterns as either MDI or DMI, with similar proportions for each type. In our study, $73 \%$ of patients were characterized, with $43 \%$ of $\mathrm{MDI}$ and $30 \%$ of DMI. Whether ethnic or geographic latitude differences exist regarding the major course patterns of BD requires further investigation.

There are some limitations to the present study. First, the convenient sampling method may have incurred selection bias; thus, caution should be exercised when generalizing these results. Second, the cross-sectional design of the study means that no long-term prescription trend could be explored. Third, the number of patients enrolled from each country varied, and samples were not demographically representative. Furthermore, the sample was ethnically diverse. Nevertheless, the present study revealed a high proportion of antipsychotic use and a high rate of polypharmacy among patients with BD in Asia. Further 
data from different countries would be needed to explore prescription trends worldwide.

In conclusion this survey delineated the different prescription patterns of pharmacotherapy on BD in 14 Asian countries. We proposed a new definition of polypharmacy for bipolar disorder for easier comparison between different study sites and course phases. Our results of different prescription patterns in specific course phases may offer a reference for clinical practitioners.

\section{- Funding}

None.

\section{Acknowledgments}

The authors thank Mr Da-Yi Tsai for Internet server maintenance and Mr Yan-Lung Chiou and Miss Li-Hung Chen for their assistance in data management. The authors are grateful to the following clinicians involved in the data collection: Byung-Joo Ham, Joon Hyuk Park, Jung Goo Lee, Seon-Cheol Park, Seung-Gon Kim, Kiwon Kim, and Euihyeon Na in Korea; Yunshu Zhang in China; Chun-Hsin Chen, Tsung-Ming Hu, and Chih-Ken Chen in Taiwan; Mazhar Malik, Imtiaz Dogar, Moin Ansari, and Mian Mukhtar ul Haq Azeemi in Pakistan; Roy Abraham in India; Lee Chung Wah, Ng Boon Seng, Syarifah Hafizah, Siti Salwa, Sit Nor Fadhlina, Chin Loi Fei, Ruzita Jamaluddin, Selva Ratnasingam, and Ng Choon Guan in Malaysia; Takashi Yoshio, Chika Kubota, Toshiya Inada, and Nobutomo Yamamoto in Japan; Chonnakarn Jatchavala in Thailand; Isa Multazam and Eunice Najoan in Indonesia.

\section{Conflicts of Interest}

No potential conflict of interest relevant to this article was reported.

\section{Author Contributions}

Designed the study, analyzed and interpreted data: Shih-Ku Lin, Shu-Yu Yang, and Naotaka Shinfuku. Data acquisition: All listed authors. Supervision: Mian-Yoon Chong and Norman Sartorius. Draft writing: Shih-Ku Lin. All authors have read and approved the revised manuscript.

\section{ORCID}

Shih-Ku Lin

https://orcid.org/0000-0002-5123-0389

Shu-Yu Yang
Seon-Cheol Park https://orcid.org/0000-0003-3691-4624 Ok-Jin Jang https://orcid.org/0000-0002-6272-7825 Yu-Tao Xiang https://orcid.org/0000-0002-2906-0029 Wen-Chen Ouyang https://orcid.org/0000-0002-0409-7888 Afzal Javed https://orcid.org/0000-0003-2518-0688 M. Nasar Sayeed Khan

https://orcid.org/0000-0001-9102-5024

Sandeep Grover https://orcid.org/0000-0002-2714-2055

Ajit Avasthi https://orcid.org/0000-0001-9718-4181

Roy Abraham Kallivayalil https://orcid.org/0000-0002-1991-3796

Kok Yoon Chee https://orcid.org/0000-0002-5755-9572

Takahiro A. Kato https://orcid.org/0000-0001-5169-2930

Kohei Hayakawa https://orcid.org/0000-0003-4594-2424

Pornjira Pariwatcharakul

https://orcid.org/0000-0003-0228-3043

Margarita Maramis https://orcid.org/0000-0001-8898-5470

Lakmi Seneviratne https://orcid.org/0000-0002-5563-0435

Sim Kang https://orcid.org/0000-0003-3209-9626

Wai Kwong Tang https://orcid.org/0000-0002-2616-6864

Tin Oo https://orcid.org/0000-0001-5405-8994

Norman Sartorius https://orcid.org/0000-0001-8708-6289

Chay-Hoon Tan https://orcid.org/0000-0002-6399-0668

Mian-Yoon Chong https://orcid.org/0000-0001-5836-0509

Yong Chon Park https://orcid.org/0000-0002-3019-5748

Naotaka Shinfuku https://orcid.org/0000-0002-7390-9077

\section{REFERENCES}

1. Fornaro M, Stubbs B, De Berardis D, Perna G, Valchera A, Veronese N, et al. Atypical antipsychotics in the treatment of acute bipolar depression with mixed features: a systematic review and exploratory meta-analysis of placebo-controlled clinical trials. Int J Mol Sci 2016;17:241.

2. Muneer A. The treatment of adult bipolar disorder with aripiprazole: a systematic review. Cureus 2016;8:e562.

3. Kishi T, Oya K, Iwata N. Long-acting injectable antipsychotics for prevention of relapse in bipolar disorder: a systematic review and meta-analyses of randomized controlled trials. Int J Neuropsychopharmacol 2016;19:pyw038.

4. Lehman AF, Lieberman JA, Dixon LB, McGlashan TH, Miller AL, Perkins DO, et al. Practice guideline for the treatment of patients with schizophrenia, second edition. Am J Psychiatry 2004;161(2 Suppl):1-56.

5. Parks JJ. Implementing practice guidelines: lessons from public mental health settings. J Clin Psychiatry 2007;68 Suppl 4: 45-48.

6. Daumit GL, Crum RM, Guallar E, Powe NR, Primm AB, Steinwachs DM, et al. Outpatient prescriptions for atypical antipsychotics for African Americans, Hispanics, and whites 
in the United States. Arch Gen Psychiatry 2003;60:121-128.

7. Chong MY, Tan CH, Fujii S, Yang SY, Ungvari GS, Si T, et al. Antipsychotic drug prescription for schizophrenia in East Asia: rationale for change. Psychiatry Clin Neurosci 2004;58: 61-67.

8. Galling B, Roldán A, Hagi K, Rietschel L, Walyzada F, Zheng $\mathrm{W}$, et al. Antipsychotic augmentation vs. monotherapy in schizophrenia: systematic review, meta-analysis and metaregression analysis. World Psychiatry 2017;16:77-89.

9. Lin SK. Antipsychotic polypharmacy: a dirty little secret or a fashion? Int I Neuropsychopharmacol 2020;23:125-131.

10. Fornaro M, De Berardis D, Koshy AS, Perna G, Valchera A, Vancampfort D, et al. Prevalence and clinical features associated with bipolar disorder polypharmacy: a systematic review. Neuropsychiatr Dis Treat 2016;12:719-735.

11. Davis JM. Dose equivalence of the antipsychotic drugs. J Psychiatr Res 1974;11:65-69.

12. Leucht S, Samara M, Heres S, Patel MX, Woods SW, Davis JM. Dose equivalents for second-generation antipsychotics: the minimum effective dose method. Schizophr Bull 2014;40: 314-326.

13. World Health Organization. WHO Collaborating centre for drug statistics and methodology [Internet]. Geneva: World Health Organization; 2017 [cited at 2018 May 1]. Available from: http://www. who.int/medicines/regulation/medicines-safety/ about/collab-centres-norwegian/en/.

14. Hayes J, Prah P, Nazareth I, King M, Walters K, Petersen I, et al. Prescribing trends in bipolar disorder: cohort study in the United Kingdom THIN primary care database 1995-2009. PLoS One 2011;6:e28725.

15. Olfson M, Blanco C, Liu SM, Wang S, Correll CU. National trends in the office-based treatment of children, adolescents, and adults with antipsychotics. Arch Gen Psychiatry 2012;69: 1247-1256.

16. Lähteenvuo M, Tanskanen A, Taipale H, Hoti F, Vattulainen P, Vieta $\mathrm{E}$, et al. Real-world effectiveness of pharmacologic treatments for the prevention of rehospitalization in a Finnish nationwide cohort of patients with bipolar disorder. JAMA Psychiatry 2018;75:347-355.

17. Grunze H, Vieta E, Goodwin GM, Bowden C, Licht RW, Möller HJ, et al. The World Federation of Societies of Biological Psychiatry (WFSBP) guidelines for the biological treatment of bipolar disorders: update 2012 on the long-term treatment of bipolar disorder. World I Biol Psychiatry 2013; 14:154-219.

18. Fountoulakis KN, Grunze H, Vieta E, Young A, Yatham L, Blier $\mathrm{P}$, et al. The International College of Neuro-Psychopharmacology (CINP) treatment guidelines for bipolar disorder in adults (CINP-BD-2017), part 3: the clinical guidelines. Int J Neuropsychopharmacol 2017;20:180-195.

19. Yatham LN, Kennedy SH, Parikh SV, Schaffer A, Bond DJ, Frey
$\mathrm{BN}$, et al. Canadian Network for Mood and Anxiety Treatments (CANMAT) and International Society for Bipolar Disorders (ISBD) 2018 guidelines for the management of patients with bipolar disorder. Bipolar Disord 2018;20:97-170.

20. Correll CU, Kane JM. One-year incidence rates of tardive dyskinesia in children and adolescents treated with second-generation antipsychotics: a systematic review. J Child Adolesc Psychopharmacol 2007; 17:647-656.

21. Gao K, Kemp DE, Ganocy SJ, Gajwani P, Xia G, Calabrese JR. Antipsychotic-induced extrapyramidal side effects in bipolar disorder and schizophrenia: a systematic review. J Clin Psychopharmacol 2008;28:203-209.

22. Mojtabai R, Olfson M. National trends in psychotherapy by office-based psychiatrists. Arch Gen Psychiatry 2008;65:962970.

23. Olfson M, Marcus SC. National trends in outpatient psychotherapy. Am J Psychiatry 2010;167:1456-1463.

24. Goldberg JF, Brooks JO 3rd, Kurita K, Hoblyn JC, Ghaemi SN, Perlis $\mathrm{RH}$, et al. Depressive illness burden associated with complex polypharmacy in patients with bipolar disorder: findings from the STEP-BD. I Clin Psychiatry 2009;70:155162.

25. Fung VC, Overhage LN, Sylvia LG, Reilly-Harrington NA, Kamali M, Gao K, et al. Complex polypharmacy in bipolar disorder: side effect burden, adherence, and response predictors. J Affect Disord 2019;257:17-22.

26. Jaracz J, Rudnicka ET, Bierejszyk M, Witczyk K, Raczyńska A, Nowak W, et al. The pattern of pharmacological treatment of bipolar patients discharged from psychiatric units in Poland. Pharmacol Rep 2018;70:694-698.

27. Kendall T, Morriss R, Mayo-Wilson E, Marcus E. Assessment and management of bipolar disorder: summary of updated NICE guidance. BMJ 2014;349: g5673.

28. Lyall LM, Penades N, Smith DJ. Changes in prescribing for bipolar disorder between 2009 and 2016: national-level data linkage study in Scotland. Br J Psychiatry 2019;215:415-421.

29. Haeberle A, Greil W, Russmann S, Grohmann R. Mono- and combination drug therapies in hospitalized patients with bipolar depression. Data from the European drug surveillance program AMSP. BMC Psychiatry 2012;12:153.

30. Weinstock LM, Gaudiano BA, Epstein-Lubow G, Tezanos K, Celis-Dehoyos CE, Miller IW. Medication burden in bipolar disorder: a chart review of patients at psychiatric hospital admission. Psychiatry Res 2014;216:24-30.

31. Yang SY, Chen LY, Najoan E, Kallivayalil RA, Viboonma K, Jamaluddin R, et al. Polypharmacy and psychotropic drug loading in patients with schizophrenia in Asian countries: fourth survey of Research on Asian Prescription Patterns on antipsychotics. Psychiatry Clin Neurosci 2018;72:572-579.

32. Tondo L, Vázquez GH, Baldessarini RJ. Depression and mania in bipolar disorder. Curr Neuropharmacol 2017;15:353- 358. 